\title{
Characterization and clinical relevance of PDGFRA pathway copy number variation gains across human cancers
}

\author{
Lizhu Liu ${ }^{1}$ Lihong Wu ${ }^{2} \cdot$ Dan Shan $^{1} \cdot$ Bo Han $^{1}$ (D) \\ Received: 25 October 2021 / Accepted: 22 January 2022 / Published online: 25 February 2022 \\ (c) The Author(s) 2022
}

\begin{abstract}
We investigated the copy number variation (CNV) of PDGFRA pathway across all common cancer types as well as its clinical relevance. This study included a total of 10,678 patients with pan-cancerous species involving 33 types of cancers and patient information was obtained from The Cancer Genome Atlas. According to the PDGFRA pathway CNV, all samples were divided into copy number gain (CN gain) group and No CN gain group. The analysis of loss of heterozygosity (LOH) fraction, CNV burden, tumor mutation burden (TMB), and the number of immunogenic mutations were performed, as well as the correlation analysis of PDGFRA pathway $\mathrm{CN}$ gain with tumor-related signaling pathways and tumor-infiltrating immune cell subpopulations. The results showed that CN gain of PDGFRA pathway in the cancer patients was associated with significantly shorter overall survival. The $\mathrm{CN}$ gain of PDGFRA pathway was identified as a prognostic risk factor for some tumors. $\mathrm{CN}$ gain was accompanied by an altered percentage of LOH, CNV burden, TMB, the number of immunogenic mutations were increased and tumor-infiltrating immune cell subpopulations were less. While certain tumor-related signaling pathways, such as hypoxia, cell cycle, DNA repair, and epithelial-mesenchymal transition were more enriched in the CN gain group, quiescence, and inflammation pathways were more enriched in the No CN gain group. In conclusion, PDGFRA pathway CNV gain may be a poor prognostic factor in cancer patients.
\end{abstract}

Keywords PDGFRA pathway · Copy number variation · Tumor-related signaling pathways · Tumor-infiltrating immune cells

Abbreviations
$\begin{array}{ll}\text { PDGFRA } & \text { Platelet-derived growth factor receptor alpha } \\ \text { MSC } & \text { Mesenchymal stromal cells } \\ \text { SPM } & \text { Somatic point mutations } \\ \text { CNV } & \text { Copy number variants } \\ \text { EMT } & \text { Epithelial-mesenchymal transition } \\ \text { OS } & \text { Overall survival } \\ \text { ACC } & \text { Adrenocortical carcinoma } \\ \text { KIRC } & \text { Kidney renal clear cell carcinoma } \\ \text { LUAD } & \text { Lung adenocarcinoma }\end{array}$

Communicated by Shuhua Xu.

Lizhu Liu and Lihong Wu are Co-first author.

Bo Han

bohan1964@163.com

1 Department of Oncology, The First Affiliated Hospital of Harbin Medical University, No.23 Post Street Nangang District, Harbin 150001, China

2 Genecast Biotechnology Co., Ltd, Wuxi 214104, China

$\begin{array}{ll}\text { SARC } & \text { Sarcoma } \\ \text { SKCM } & \text { Skin Cutaneous Melanoma } \\ \text { UCEC } & \text { Uterine Corpus Endometrial Carcinoma } \\ \text { LOH } & \text { Loss of heterozygosity } \\ \text { TMB } & \text { Tumor mutation burden } \\ \text { TNB } & \text { Tumor neoantigen burden } \\ \text { ssGSEA } & \text { single-sample gene set enrichment analysis } \\ \text { IQR } & \text { Interquartile variance } \\ \text { KM } & \text { Kaplan-Meier }\end{array}$

\section{Introduction}

Platelet-derived growth factor receptor alpha (PDGFRA) gene encodes a tyrosine kinase receptor that activates tyrosine kinase (Ong et al. 2018). It has been shown that $P D G$ $F R A$ is involved in gene mutation, tumor cell proliferation, migration and invasion, maintenance of mesenchymal stromal cells (MSC), and immune infiltration, while it may serve as a potential biomarker and therapeutic target (Chang et al. 2018; Pantaleo et al. 2019; Wang et al. 2020a). Activation of 
PDGFRA signaling is sufficient to drive fibrosis in diverse organs. Targeting PDGFRA signaling can be an effective approach to treat fibrosis (Decker et al. 2017). However, the role of PDGFR signaling pathway in the pan-cancer context has yet to be investigated.

Somatic mutations are closely related to immunotherapy and clinical outcomes (e.g., survival), while these mutations can be classified as somatic point mutations (SPM) or somatic copy number variants (CNV). CNVs have been shown to be widely present in normal individuals (Hieronymus et al. 2018). As an important form of genetic structural variation, CNVs result from gains or losses of DNA segments larger than $1 \mathrm{~kb}$ in the human genome (Shao et al. 2019). Recently, Zheng et al. showed that CNV data had a higher validity in predicting cancer prognosis than SPM data (Zheng et al. 2020). Furthermore, the relationship between CNVs and gene expression is crucial for the prevention, diagnosis, and treatment of cancer (Shao et al. 2019). CNV-caused genetic mutations may lead to the development of immune escape (Chen et al. 2020). It has also been reported that copy number gains ( $\mathrm{CN}$ gain) occur more frequently than copy number losses (CN loss) during epithelial-mesenchymal transition (EMT), and somatic $\mathrm{CN}$ gain activates gene expression by increasing gene dosage (Zhao et al. 2016). Interestingly, Anwar et al. identified frequent recurrence of CNVs in PDGFRA gene in a next-generation sequencing-based CNV study of invasive breast cancer patients (Anwar et al. 2020). PDGFRA CN gain was also detected in intractable epilepsy and high-grade astrocytoma and found to be significantly associated with the treatment and prognosis of the disease (Phillips et al. 2013; Vasudevaraja et al. 2021). While the importance of the tumor microenvironment has been increasingly recognized, the complexity of interaction between tumor cells and their microenvironment is becoming evident. However, characterization and clinical properties of PDGFRA CN gain in whole cancers have not been well documented.

To comprehensively characterize PDGFRA CN gain, we collected the CNV data and clinical information of pancancer patients from the TCGA database, systematically analyzed functional status of the tumor cells, and quantified the components of immune cells in the tumor immune microenvironment. In this study, we identified PDGFRA CN gain as a prognostic risk factor for a significantly shorter overall survival (OS) in six cancer species, including adrenocortical carcinoma (ACC), kidney renal clear cell carcinoma (KIRC), lung adenocarcinoma (LUAD), sarcoma (SARC), skin cutaneous melanoma (SKCM), and uterine corpus endometrial carcinoma (UCEC). Moreover, the enrichment of related pathways that promote tumor development was higher in these six cancer types. Loss of heterozygosity (LOH), CNV burden was higher and effector immune cells all affect patient prognosis.

\section{Materials and methods}

\section{Data collection and processing}

The CNV data and clinical information for 10,678 patients with pan-cancerous species were downloaded from the TCGA database (https://gdc.cancer.gov/about-data/publi cations/panimmune). The reference data for gene sets contained in PDGFRA signaling pathway were obtained from the GSEA website (https://www.gsea-msigdb.org/gsea/ msigdb/cards/PID_PDGFRA_PATHWAY.html) (Table 1). We defined the tumor with CN gain in any of PDGFRA pathway genes as having PDGFRA pathway $\mathrm{CN}$ gain. Table $\mathrm{S} 1$ shows the number of cases for each cancer type and the number of cases with PDGFRA pathway $\mathrm{CN}$ gain variants in the TCGA cohort. The tumor mutation burden (TMB), the number of immunogenic mutations (TNB), CNV burden score, and LOH score were derived from the published studies (Thorsson et al. 2018).

\section{Assessment of the functional status of tumor cells}

To assess the functional status of tumor cells, 14 manually curated cancer-related signatures were calculated using the GSVA package in R software, including stemness, invasion,

Table 1 Gene list in PDGFRA pathway

\begin{tabular}{lll}
\hline Gene symbol & Ensembl Gene ID & Gene ID \\
\hline FOS & ENSG00000170345 & 2353 \\
IFNG & ENSG00000111537 & 3458 \\
JUN & ENSG00000177606 & 3725 \\
ITGAV & ENSG00000138448 & 3685 \\
SRF & ENSG00000112658 & 6722 \\
PDGFRA & ENSG00000134853 & 5156 \\
PLCG1 & ENSG00000124181 & 5335 \\
ELK1 & ENSG00000126767 & 2002 \\
JAK1 & ENSG00000162434 & 3716 \\
PIK3R1 & ENSG00000145675 & 5295 \\
SHC1 & ENSG00000160691 & 6464 \\
PIK3CA & ENSG00000121879 & 5290 \\
CRK & ENSG00000167193 & 1398 \\
CRKL & ENSG00000099942 & 1399 \\
CAV3 & ENSG00000182533 & 859 \\
GRB2 & ENSG00000177885 & 2885 \\
CSNK2A1 & ENSG00000101266 & 1457 \\
CAV1 & ENSG00000105974 & 857 \\
SOS1 & ENSG00000115904 & 6654 \\
RAPGEF1 & ENSG00000107263 & 2889 \\
SHB & ENSG00000107338 & 6461 \\
SHF & ENSG00000138606 & 90,525 \\
\hline
\end{tabular}


metastasis, proliferation, EMT, angiogenesis, apoptosis, cell cycle, differentiation, DNA damage, DNA repair, hypoxia, inflammation, and quiescence (Yuan et al. 2019).

\section{Quantitative analysis of tumor-infiltrating immune cell subpopulations}

To quantify the components of immune cells in the tumor microenvironment, the enrichment of 28 immune cell subpopulations was assessed using the single-sample gene set enrichment analysis (ssGSEA) method (Angelova et al. 2015).

\section{Statistical analysis}

All statistical analyses were performed using $\mathrm{R}$ software (version 3.4.2). Data were presented as median and interquartile variance (IQR). The Student's $t$ test and Wilcoxon rank-sum test were used to analyze the differences between the two groups for normally distributed data and non-normally distributed data, respectively. Survival data were analyzed using Kaplan-Meier (KM) survival curves and log-rank test. The relationship between PDGFRA pathway $\mathrm{CN}$ gain in pan-cancer species and OS was examined using univariate COX regression. Bilateral $P$ values $<0.05$ were considered statistically significant.

\section{Results}

\section{The CNVs of PDGFRA pathway in pan-cancerous species}

We analyzed the CNVs of each PDGFRA pathway gene in TCGA database for each cancer type and found that the frequency of $\mathrm{CN}$ gain or $\mathrm{CN}$ loss in PDGFRA pathway varied from one cancer type to another (Fig S1). As illustrated in Fig. 1A, the frequency of CN gains in PDGFRA pathway ranged from 1 to $45 \%$ among various cancer types. Moreover, survival analysis showed that patients with $\mathrm{CN}$ gain in PDGFRA pathway had significantly shorter OS and worse prognosis $(P<0.0001)$ than the No $\mathrm{CN}$ gain group (Fig. 1B), whereas no significant correlation between $\mathrm{CN}$ loss in PDGFRA pathway and OS was found (Fig. 1C). Notably, univariate analysis identified PDGFRA pathway $\mathrm{CN}$ gain as a risk factor for poor OS in the following six cancer types: ACC $(P<0.05)$, KIRC $(P<0.01)$, LUAD $(P<0.05)$, SARC $(P<0.05)$, SKCM $(P<0.05)$, and UCEC $(P<0.001)$ (Fig. 1D).

We next performed KM survival analysis and log-rank test to verify the relationships between the $\mathrm{CN}$ gain and survival rates of the above-mentioned six cancer types. As shown in Fig. 2, OS was significantly shorter in patients with the CN gain of PDGFRA pathway for all six cancer types compared with the No $\mathrm{CN}$ gain group $(P<0.05)$.

\section{Analysis of PDGFRA pathway CN gain in relation to LOH fraction, CNV burden, TMB, and TNB}

Based on whether a CN gain in PDGFRA pathway was identified, patients in this study were divided into two groups: $\mathrm{CN}$ gain and No $\mathrm{CN}$ gain. We further analyzed the differences in LOH fraction, CNV burden, TMB, and TNB between the two groups of patients, and found that the differences were significant $(P<0.05)$ (Fig S2 and Table S2). Moreover, we observed that the $\mathrm{CN}$ gain of PDGFRA pathway was associated with a higher proportion of $\mathrm{LOH}$ alterations and higher $\mathrm{CNV}$ burden in most cancers such as KIRC, LUAD, SKCM, and UCEC, while the association was in the opposite direction in ACC (Fig. 3A, B). Likewise, while association of the $\mathrm{CN}$ gain in PDGFRA pathway with higher TMB and TNB values was identified in ACC, LUAD and some other types of cancers, the association was in the opposite direction in UCEC (Fig. 3C,D).

\section{Relationships between the CN gain of PDGFRA pathway and tumor-related signaling pathways}

Next, we examined the relationships between the $\mathrm{CN}$ gain of PDGFRA pathway and 14 tumor-related signaling pathways in the pan-cancer species (Fig S3). As depicted in Fig. 4A-D, F, in ACC, KIRC, LUAD, SARC, and UCEC, pathways related to tumorigenic progression, including hypoxia, cell cycle, DNA repair, and EMT, were more enriched in the $\mathrm{CN}$ gain group as compared to No $\mathrm{CN}$ gain group. On the contrary, in SKCM, pathways such as quiescence and inflammation were more enriched in the No CN gain group (Fig. 4E).

\section{Relationships between the CN gain of PDGFRA pathway and tumor-infiltrating immune cell subsets}

Furthermore, we investigated the relationships between the CN gain of PDGFRA pathway and tumor-infiltrating immune cell subpopulations in pan-cancerous species (Fig. S4). Compared with the $\mathrm{CN}$ gain group, tumor-infiltrating immune cell subpopulations were more abundant in the No $\mathrm{CN}$ gain group among many cancer types. Thereafter, we analyzed the difference in the enrichment of tumor-infiltrating immune cell subpopulations between the $\mathrm{CN}$ gain and No $\mathrm{CN}$ gain groups among the six cancer species in which the CN gain of PDGFRA pathway was significantly associated with OS. The analysis revealed that in ACC, three immune cell subsets, Neutrophil, Eosinophil, and Natural killer cell, were significantly more abundant in the $\mathrm{CN}$ gain group (Fig. 5A), while in SARC, MDSC, Macrophage, 
A

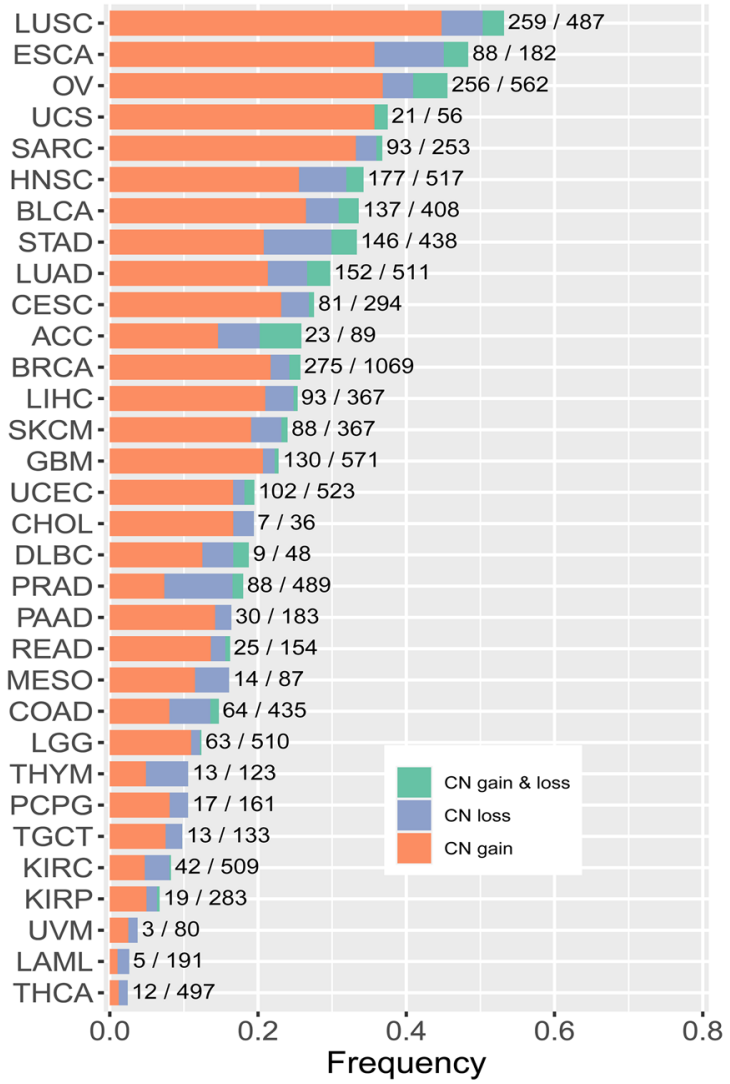

B

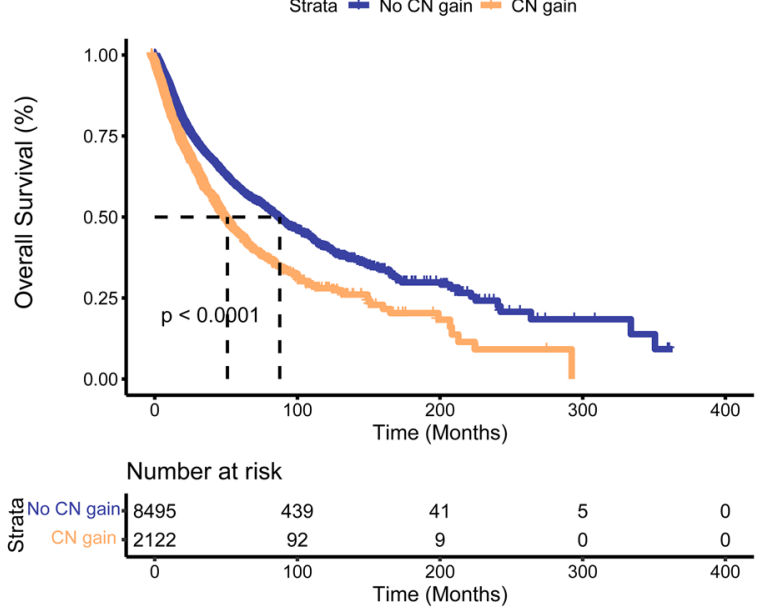

Central memory CD8 T cell, Regulatory T cell, Activated CD8 T cell and other immune cell subsets were significantly more enriched in the $\mathrm{CN}$ gain group (Fig. 5D). Conversely, in KIRC and SKCM, several immune cell subsets such as Immature B cell, Activated B cell, Effector memory CD8 T cell, and Type I T helper cell were significantly more abundant in the No CN gain group (Fig. 5B, E). Notably, we observed that in LUAD and UCEC, there were significant differences in the enrichment of certain immune cell subsets
D

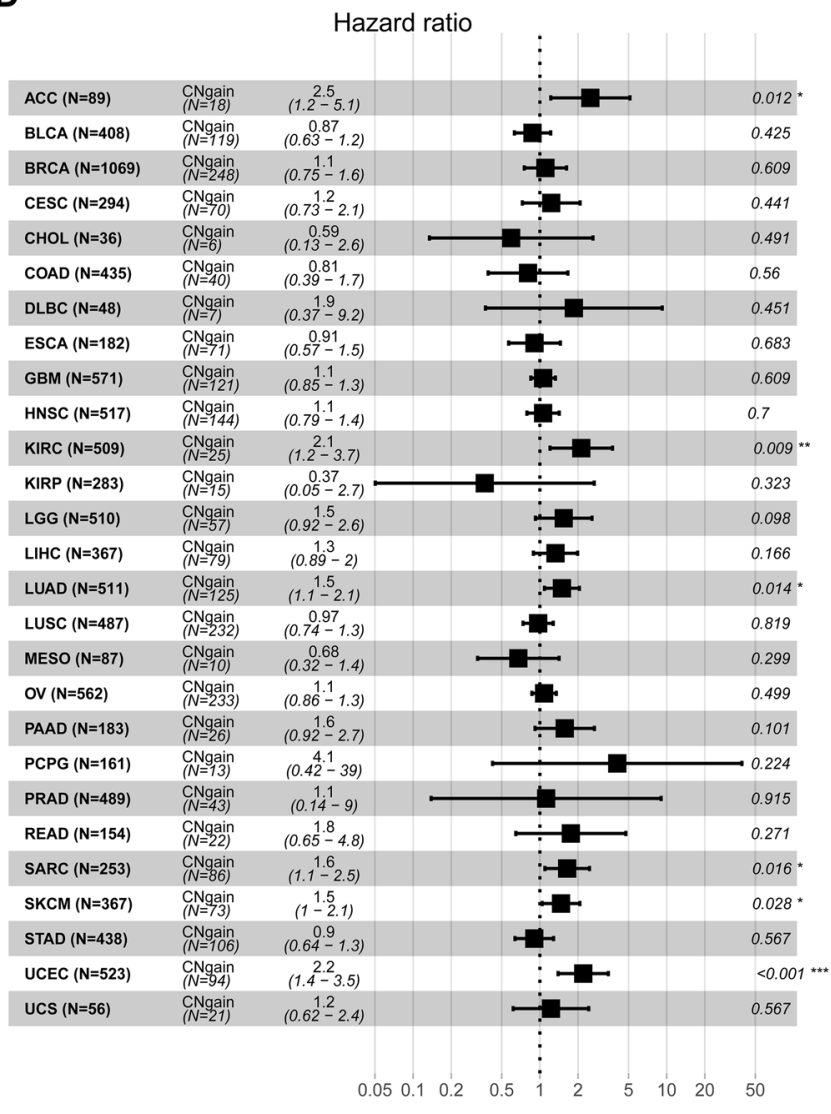

C

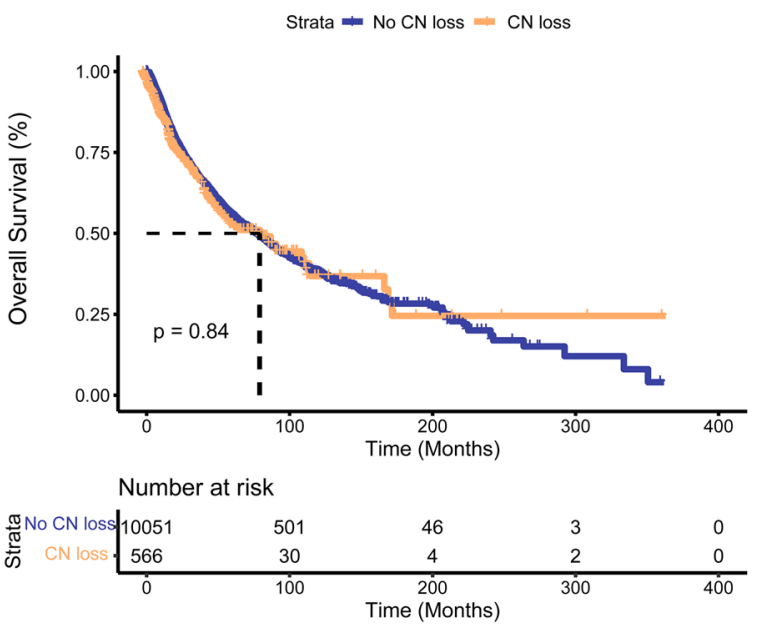

such as Eosinophil between the $\mathrm{CN}$ gain and No $\mathrm{CN}$ gain groups (Fig. 5C, F).

\section{Discussion}

PDGFRA is of critical importance in mesenchymal development, homeostasis and pathogenesis. Aberrant PDGFRA activities have been linked to a variety of diseases, including 
4Fig. 1 Relationships between the CN gain of PDGFRA pathway and prognosis of pan-cancer species. A Frequency of CNVs in PDGFRA pathway among different cancer species. The CNVs were classified as $\mathrm{CN}$ gain, $\mathrm{CN}$ loss, or $\mathrm{CN}$ gain \& loss. B KM survival analysis of the relationship between the $\mathrm{CN}$ gain of PDGFRA pathway and OS in all pan-cancer samples. C KM survival analysis of the relationship between the CN loss of PDGFRA pathway and OS in all pancancer samples. D One-way COX regression analysis of the relationship between the $\mathrm{CN}$ gain and $\mathrm{OS}$ in various cancer species. $P<0.05$ was considered as a significant difference. ${ }^{*} P<0.05$, $* * P<0.01$, and $* * * P<0.001$. ACC adrenocortical carcinoma; $B L C A$ bladder urothelial carcinoma; $B R C A$ breast invasive carcinoma; $C E S C$ cervical squamous cell carcinoma and endocervical adenocarcinoma; CHOL cholangiocarcinoma; $C O A D$ colon adenocarcinoma; $D L B C$ lymphoid neoplasm diffuse large b-cell lymphoma; ESCA esophageal carcinoma; GBM glioblastoma multiforme; HNSC head and neck squamous cell carcinoma; KICH kidney chromophobe; KIRC kidney renal clear cell carcinoma; KIRP kidney renal papillary cell carcinoma; $L A M L$ acute myeloid leukemia; $L G G$ brain lower grade glioma; $L I H C$ liver hepatocellular carcinoma; $L U A D$ lung adenocarcinoma; LUSC lung squamous cell carcinoma; MESO mesothelioma; $O V$ ovarian serous cystadenocarcinoma; $P A A D$ pancreatic adenocarcinoma; $P C P G$ pheochromocytoma and paraganglioma; $P R A D$ prostate adenocarcinoma; $R E A D$ rectum adenocarcinoma; $S A R C$ sarcoma; $S K C M$ skin cutaneous melanoma; STAD stomach adenocarcinoma; TGCT testicular germ cell tumors; THCA thyroid carcinoma; THYM thymoma; UCEC uterine corpus endometrial carcinoma; UCS uterine carcinosarcoma; $U V M$ uveal melanoma

fibrosis, cancer, and pediatric diseases (Mueller et al. 2016; Decker et al. 2017). In this study, we analyzed the distribution of PDGFRA pathway CN gain in pan-cancer patients based on information from the TCGA database and investigated the association of PDGFRA pathway $\mathrm{CN}$ gain with tumorigenesis-related pathways, immune cell subpopulations, and survival rates.

First, we showed that the frequency of $\mathrm{CN}$ gain or $\mathrm{CN}$ loss in PDGFRA pathway varied among different cancer types. Notably, the CN gain of PDGFRA pathway was significantly associated with shorter OS in six cancer types including ACC, KIRC, LUAD, SARC, SKCM, and UCEC, thus serving as a prognostic risk factor for poor OS. It has been shown that $\mathrm{CN}$ gain at the PDGFRA locus can influence the relative frequency of tumor malignant cells and promote the tumor growth, whereas high expression of PDGFRA in glioblastoma can markedly improve the prognosis of patients (Motomura et al. 2012; Neftel et al. 2019). Combined with the previous studies, the findings in this study laterally validated the notion that signaling pathways can be selected during tumorigenesis in relation to certain cellular states during normal development and play a role in stabilizing specific malignant cellular states. $\mathrm{LOH}$ fraction, $\mathrm{CNV}$ burden, TMB, and TNB are the common types of cancer-causing mutations (Liu et al. 2019; Sokol et al. 2020). Here, we observed an increase in LOH (immune escape), CNV burden enrichment, TMB and TNB values in most of the cancer types with the CN gain of PDGFRA pathway, except in ACC and UCEC, suggesting a new research direction for ACC and UCEC.
Next, we examined the relationships between the $\mathrm{CN}$ gain of PDGFRA pathway and 14 tumor-related signaling pathways in each cancer type, especially the above-mentioned six cancer types with poor OS. The study revealed that there was a significant enrichment of pathways related to tumorigenic progression, such as Hypoxia, cell cycle, DNA repair, and EMT in the group of PDGFRA pathway $\mathrm{CN}$ gain. Hypoxia pathway is critical for the function of cells, organs and organisms. Liu et al. showed that both Hypoxia and PDGFRA amplification were associated with tumor localization and could serve as potential targets for specific therapies (Liu et al. 2016). While cell cycle plays an important regulatory role in diseases, small molecule inhibitors regulating the cell cycle can cause PDGFRA overexpression and CNV imbalance (Paugh et al. 2011). DNA repair is a key system for identifying and repairing structural or sequence abnormalities in DNA. The combination of PDGFR pathway, CNV, and DNA repair analysis can identify potential therapeutic targets (Zarghooni et al. 2010). Activation of EMT provides cancer cells with increased plasticity that is required for their invasion and metastasis. Studies have shown that PDGFRA activates the EMT pathway and decreases the expression of genes that favor epithelial integrity, enhancing metastatic diseases (Lopez-Campistrous et al. 2020). In the present study, we observed that in SKCM, quiescence and inflammation pathways were more enriched in the No $\mathrm{CN}$ gain group. This observation was consistent with the previous reports (Moon et al. 2017). Given that both PDGFRA CNVs and dysregulation of tumor-related pathways are usually present in cancer, we propose that the CN gain of PDGFRA pathway could be a potential target for analyzing cancer-related mechanisms in ACC, KIRC, LUAD, SARC, and UCEC.

Moreover, we found that in most cancers, tumor-infiltrating immune cell subsets, especially the effector immune cell subsets such as Activated CD8 T cell, Effector memory CD8 T cell, Activated B cell, and Immature B cell are less abundant in the group of PDGFRA pathway $\mathrm{CN}$ gain (Seo et al. 2018; Han et al. 2020; Bian et al. 2021). This observation may underlie the worse prognosis linked to the $\mathrm{CN}$ gain in PDGFRA pathway. On the contrary, in ACC and SARC, more tumor-infiltrating immune cell subsets were enriched in the $\mathrm{CN}$ gain group; among them, the dominant ones were pro-tumor growth cell subsets such as Neutrophil, Eosinophil, MDSC, and Macrophage. It has been shown that PDGFRA induces proliferation and differentiation of eosinophils and neutrophils, while $\mathrm{CN}$ gain can cause dysregulation of the two immune cell subsets (Buitenhuis et al. 2007; Wang et al. 2020b). In contrast, the effector immune cell subpopulations including Immature B cells and Activated B cells were more enriched in No CN gain group of PDGFRA pathway, particularly in KIRC and SKCM. Taken together, these data indicate that the tumor immune microenvironment varies among the cancer types and is related to $\mathrm{CNV}$ 
A

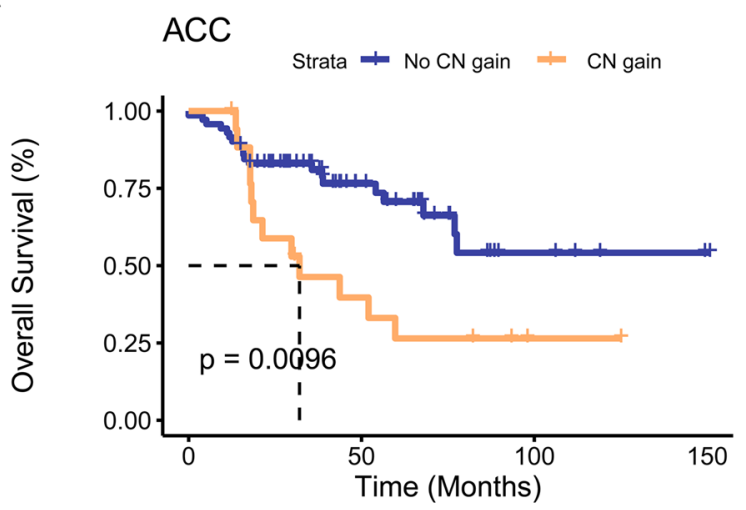

Number at risk

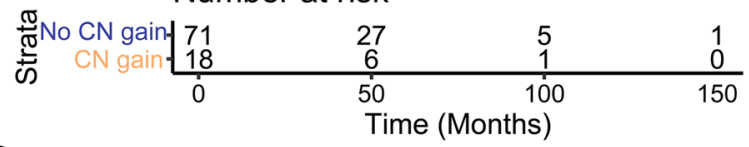

C
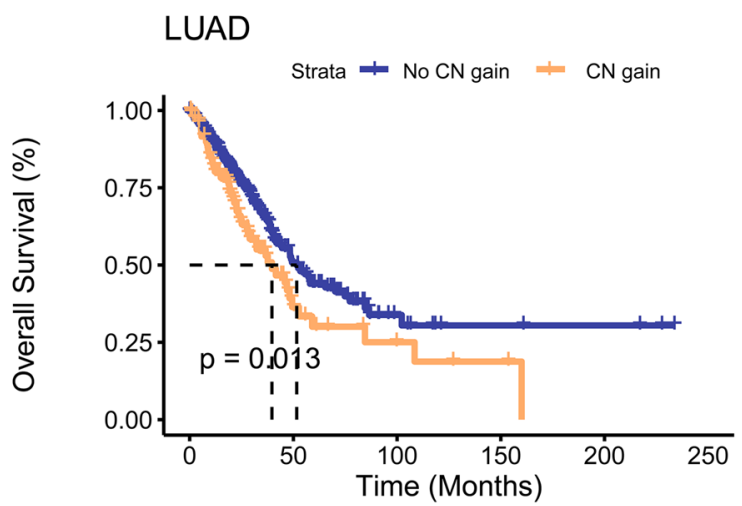

Number at risk

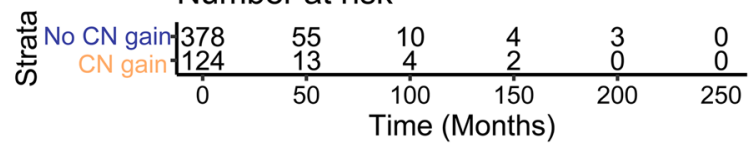

$\mathbf{E}$

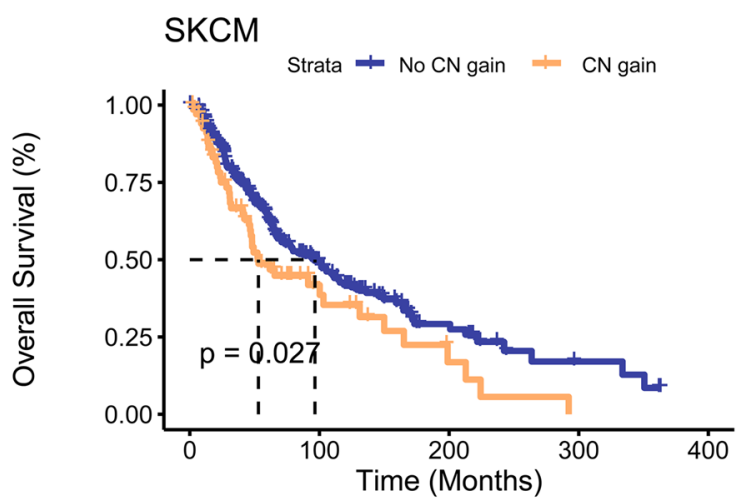

Number at risk

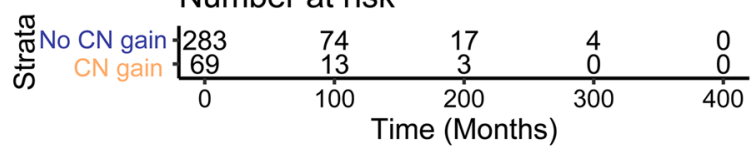

Fig. 2 Relationship between the CN gain of PDGFRA pathway and prognosis of the six cancer types. KM survival curves showing the relationship between the $\mathrm{CN}$ gain of PDGFRA pathway and OS in six
B

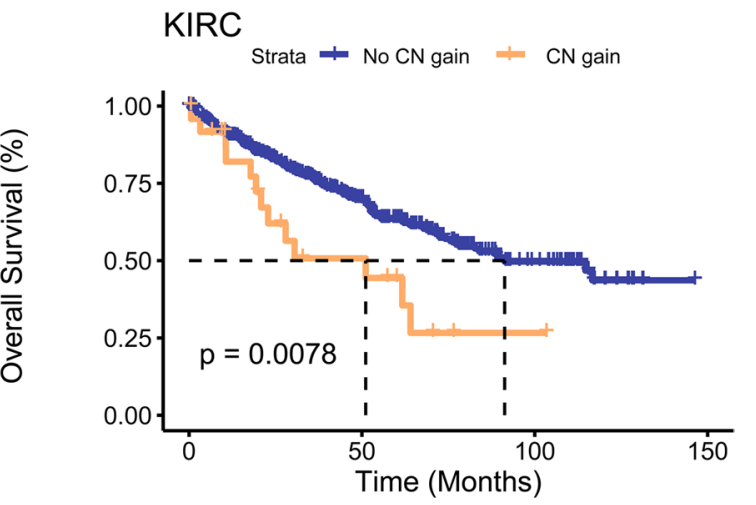

Number at risk

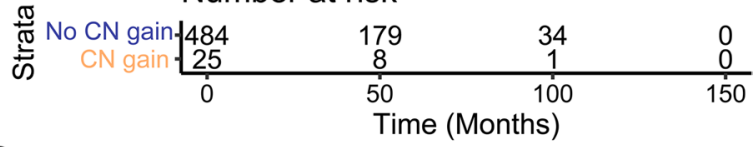

D

\section{SARC}

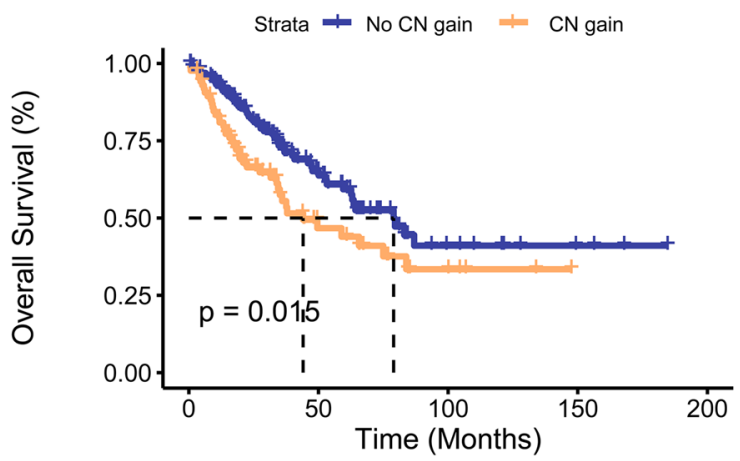

Number at risk

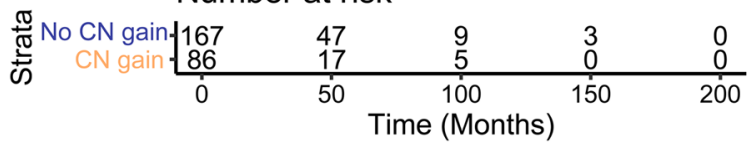

$\mathbf{F}$

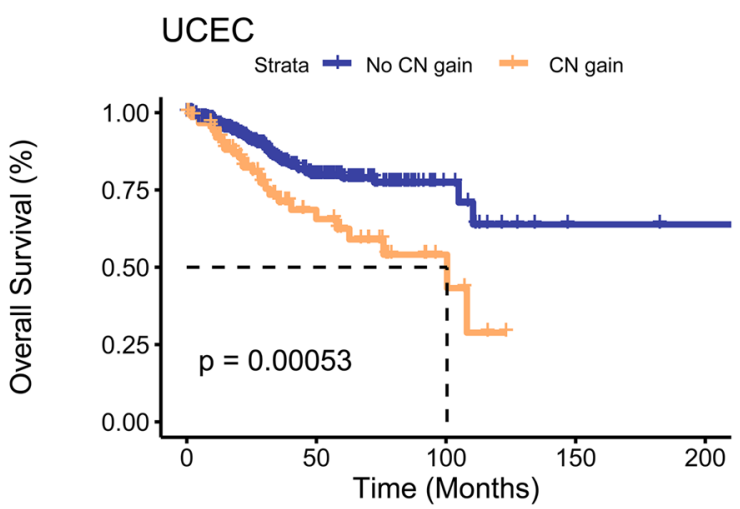

Number at risk

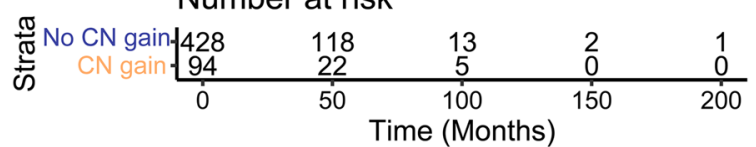

cancer types including ACC (A), KIRC (B), LUAD (C), SARC (D), $\operatorname{SKCM}(\mathbf{E})$, and $\operatorname{UCEC}(\mathbf{F})$ 
Fig. 3 Relationships of PDGFRA pathway $\mathrm{CN}$ gain with LOH fraction, CNV burden, TMB, and TNB in pan-cancerous species. Comparison of LOH fraction $(A), \mathrm{CNV}$ burden (B), TMB (non-silence per MB) (C), and TNB (number of immunogenic mutation) (D) in different cancer species between the pathway $\mathrm{CN}$ gain and No $\mathrm{CN}$ gain groups
A

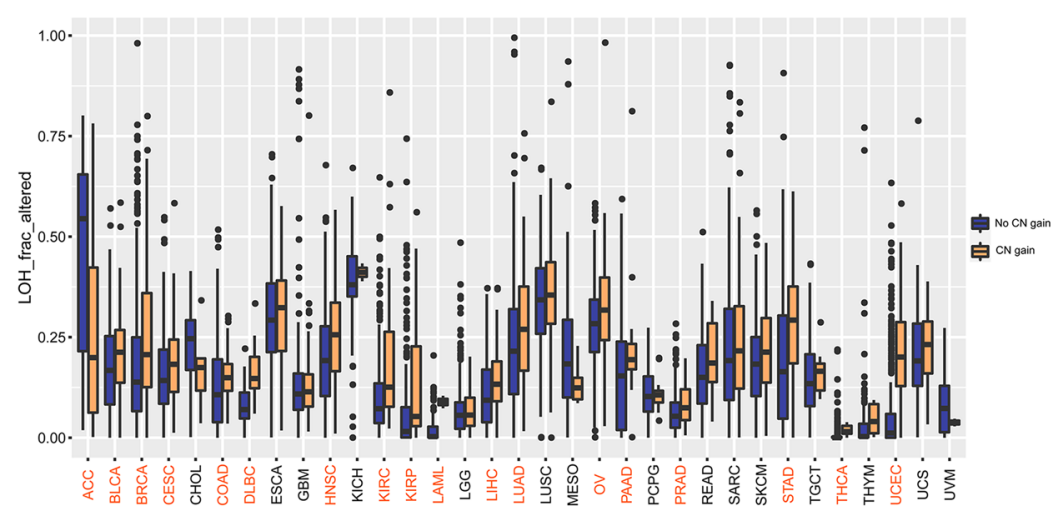

B

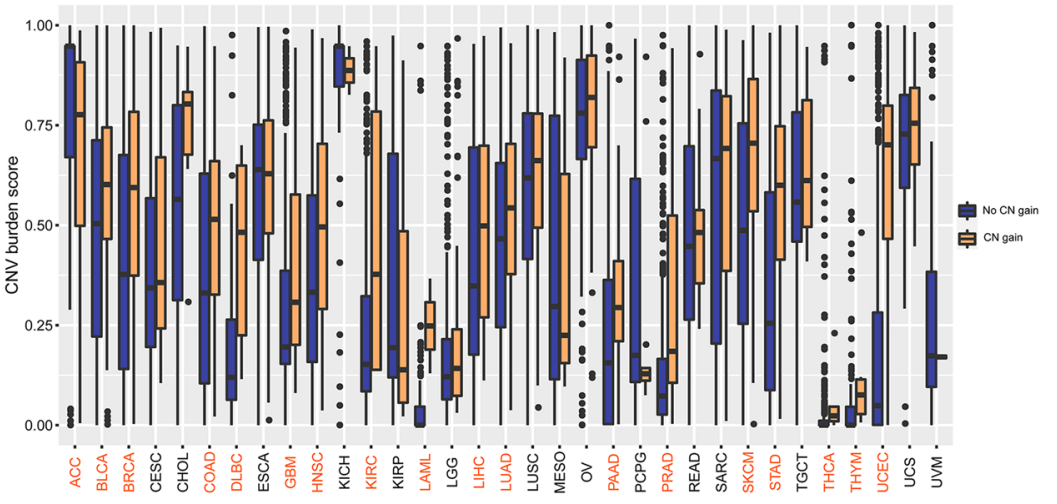

C

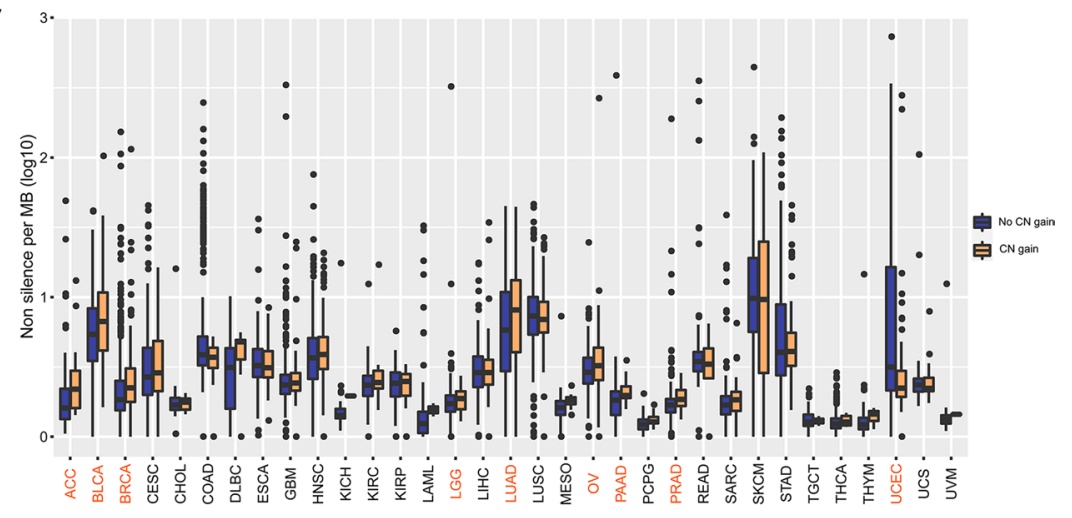

D

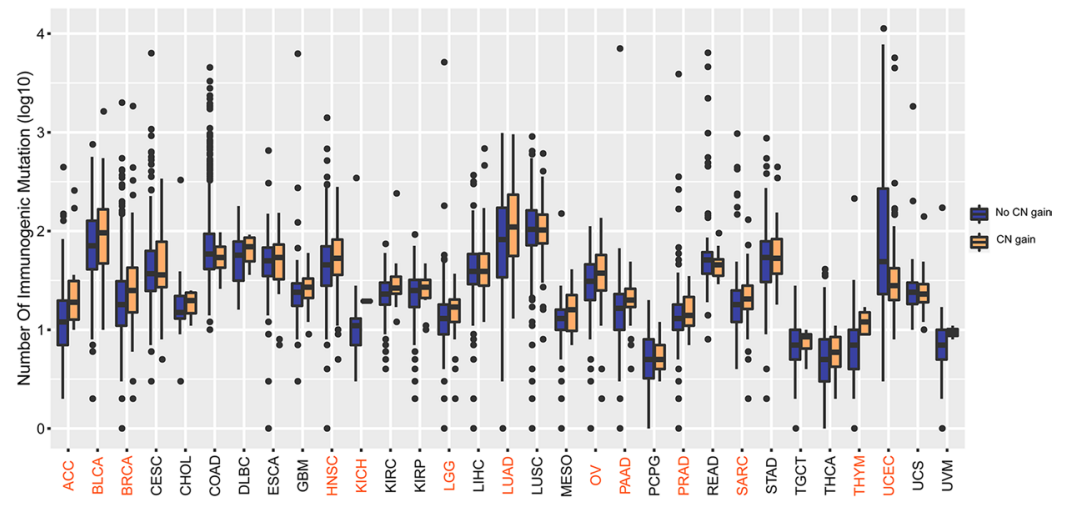


A

ACC

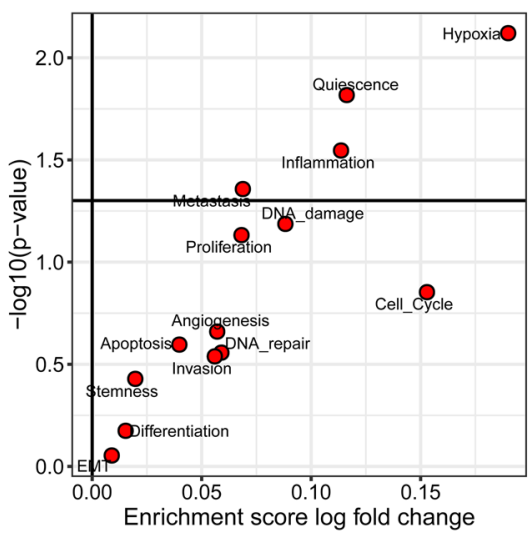

D

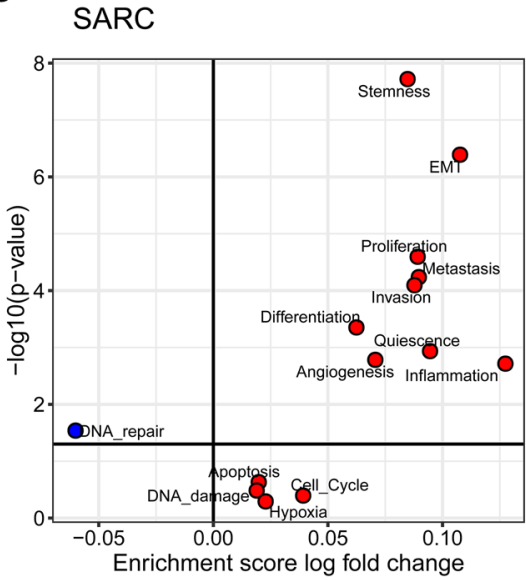

B

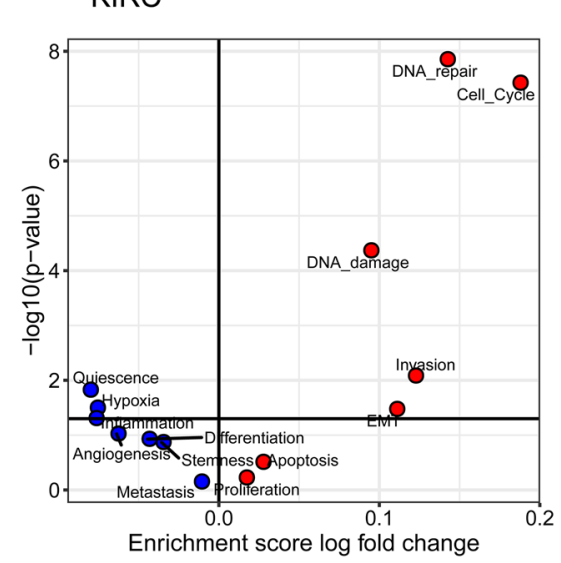

$\mathbf{E}$

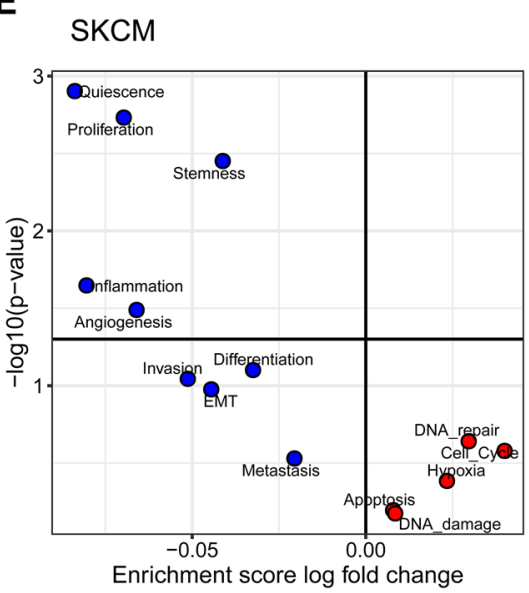

C

LUAD

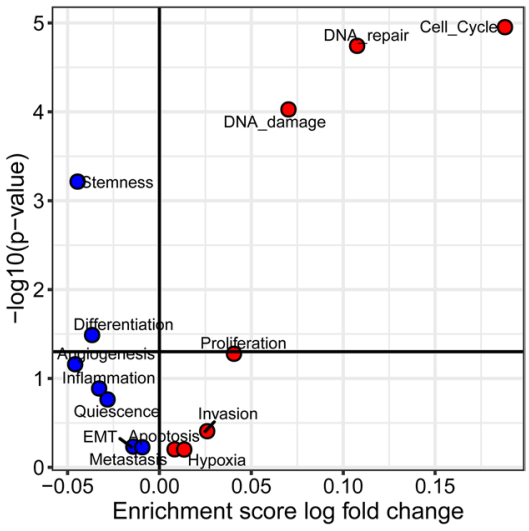

$\mathbf{F}$

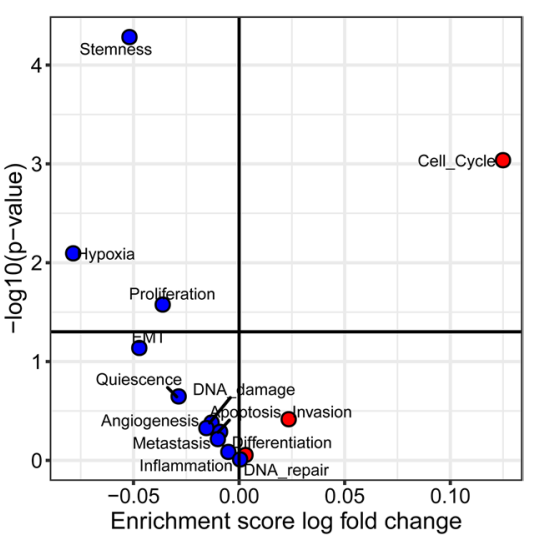

Fig. 4 Relationships between the CN gain of PDGFRA pathway and tumor-related signaling pathways in pan-cancer species. The relationships between the $\mathrm{CN}$ gain of PDGFRA pathway and 14 tumorrelated signaling pathways in six cancer species with significant survival, including ACC (A), KIRC (B), LUAD (C), SARC (D), SKCM
$(\mathbf{E})$, and UCEC $(\mathbf{F})$. Note that the upper part and lower part of the horizontal divider indicate $P<0.05$ and $P>0.05$, respectively. The left blue dot and right red dot of the vertical divider represent $\mathrm{CN}$ gain/No $\mathrm{CN}$ gain $<1$ and $\mathrm{CN}$ gain/No $\mathrm{CN}$ gain $>1$, respectively

specific mechanisms underlying the $\mathrm{CN}$ gain of PDGFRA pathway and the above three cancer types remain to be further investigated. In SARC, the CN gain of PDGFRA pathway promoted tumorigenesis. The tumor-related pathways and immune cell subpopulations were relatively enriched, but the immune cells had weaker effects, resulting in worse survival rate. $\mathrm{CNV}$ is an important event in the development of SARC and is associated with dysregulation of PDGFRA, indicating a poor prognosis (Helbig et al. 2017). This observation was consistent with the OS data obtained in the present study. Similarly, we showed that in SKCM, the CN gain of PDGFRA pathway caused a lower enrichment of multiple immune cell subpopulations, resulting in worse survival. It has been demonstrated that while the immune microenvironment is critical to the treatment of SKCM patients, CNV has the potential of being a biomarker for active melanoma disease and 
A

ACC

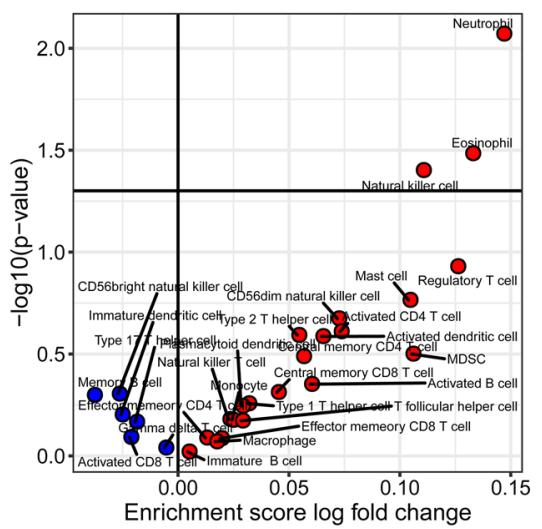

D

SARC

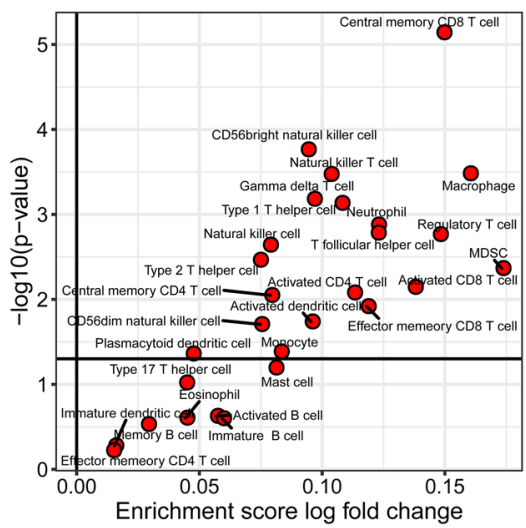

B

KIRC

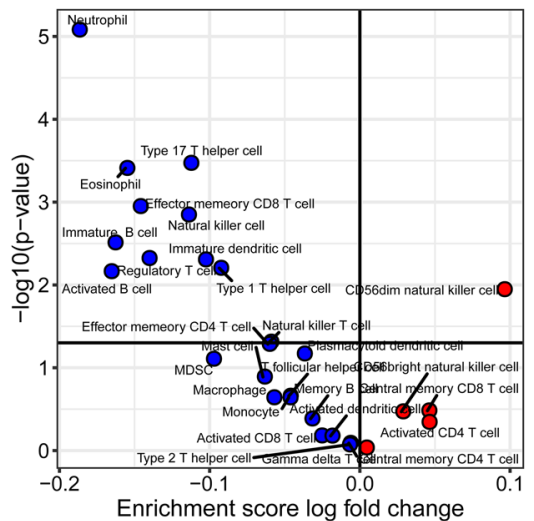

E

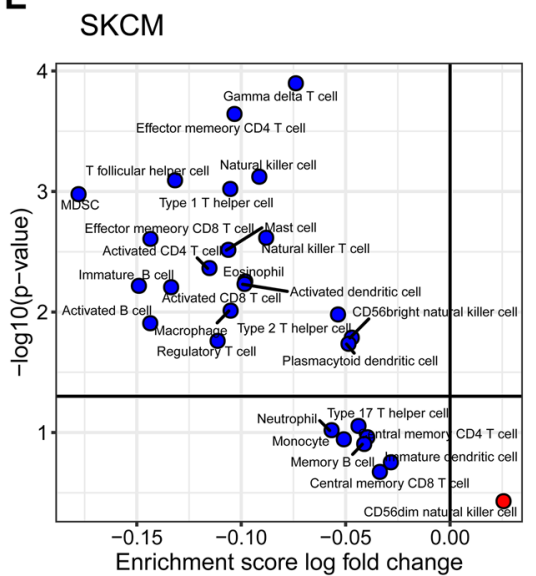

C

LUAD

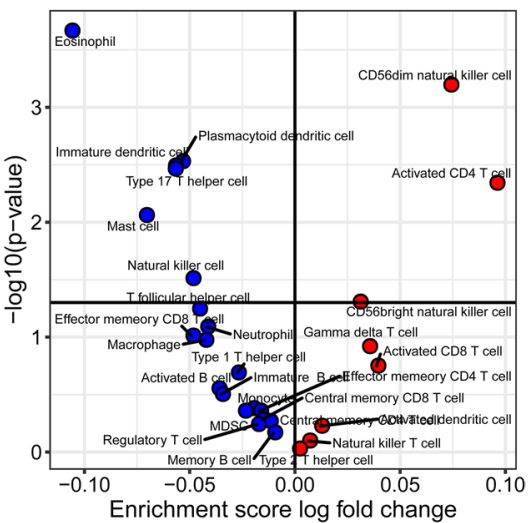

$\mathbf{F}$

UCEC

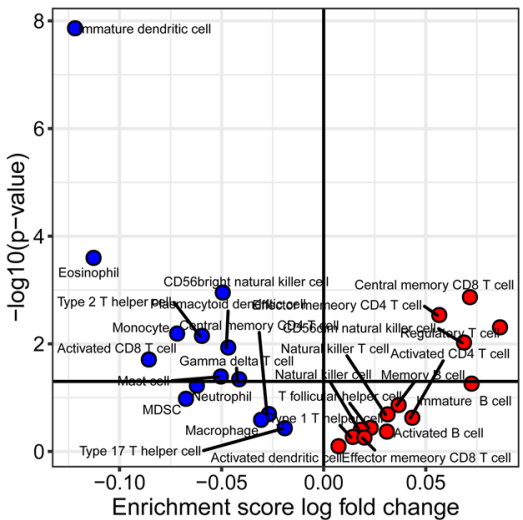

Fig. 5 Relationships between the CN gain of PDGFRA pathway and tumor-infiltrating immune cell subpopulations in pan-cancerous species. The relationships between the $\mathrm{CN}$ gain of PDGFRA pathway and tumor-infiltrating immune cell subpopulations in six cancer species with significant survival, including ACC (A), KIRC (B), LUAD
(C), SARC (D), SKCM (E), and UCEC (F). The upper part and lower part of the horizontal divider indicate $P<0.05$ and $P>0.05$, respectively. The left blue dot and right red dot of the vertical divider represent $\mathrm{CN}$ gain/No $\mathrm{CN}$ gain $<1$ and $\mathrm{CN}$ gain/No $\mathrm{CN}$ gain $>1$, respectively

Supplementary Information The online version contains supplementary material available at https://doi.org/10.1007/s00438-022-01860-y.

Author contributions LL resources, validation, writing —original draft. LW resources, validation, writing - original draft. DS visualization, formal analysis. BH conceptualization, methodology, supervision, writing - review \& editing. All authors have read and approved the final version.

Funding Not applicable.

Data availability The datasets used during the current study available from the corresponding author on reasonable request.

Code availability Not applicable.

\section{Declarations}

Conflict of interest The authors declare that they are no conflict of interests. 
Ethics approval Not applicable.

Consent to participate Not applicable.

Consent for publication Not applicable.

Open Access This article is licensed under a Creative Commons Attribution 4.0 International License, which permits use, sharing, adaptation, distribution and reproduction in any medium or format, as long as you give appropriate credit to the original author(s) and the source, provide a link to the Creative Commons licence, and indicate if changes were made. The images or other third party material in this article are included in the article's Creative Commons licence, unless indicated otherwise in a credit line to the material. If material is not included in the article's Creative Commons licence and your intended use is not permitted by statutory regulation or exceeds the permitted use, you will need to obtain permission directly from the copyright holder. To view a copy of this licence, visit http://creativecommons.org/licenses/by/4.0/.

\section{References}

Angelova M, Charoentong P, Hackl H et al (2015) Characterization of the immunophenotypes and antigenomes of colorectal cancers reveals distinct tumor escape mechanisms and novel targets for immunotherapy. Genome Biol 16:64. https://doi.org/10.1186/ s13059-015-0620-6

Anwar T, Rufail ML, Djomehri SI et al (2020) Next-generation sequencing identifies recurrent copy number variations in invasive breast carcinomas from Ghana. Mod Pathol 33:1537-1545. https://doi.org/10.1038/s41379-020-0515-2

Bian T, Zheng M, Jiang D et al (2021) Prognostic biomarker TUBA1C is correlated to immune cell infiltration in the tumor microenvironment of lung adenocarcinoma. Cancer Cell Int 21:144. https:// doi.org/10.1186/s12935-021-01849-4

Buitenhuis M, Verhagen LP, Cools J et al (2007) Molecular mechanisms underlying FIP1L1-PDGFRA-mediated myeloproliferation. Cancer Res 67:3759-3766. https://doi.org/10.1158/0008-5472. CAN-06-4183

Chang KK, Yoon C, Yi BC et al (2018) Platelet-derived growth factor receptor-alpha and -beta promote cancer stem cell phenotypes in sarcomas. Oncogenesis 7:47. https://doi.org/10.1038/ s41389-018-0059-1

Chen X, Lin Y, Qu Q et al (2020) A multi-source data fusion framework for revealing the regulatory mechanism of breast cancer immune evasion. Front Genet 11:595324. https://doi.org/10.3389/ fgene. 2020.595324

Decker M, Martinez-Morentin L, Wang G et al (2017) Leptin-receptorexpressing bone marrow stromal cells are myofibroblasts in primary myelofibrosis. Nat Cell Biol 19:677-688. https://doi.org/ 10.1038/ncb3530

Han J, Khatwani N, Searles TG et al (2020) Memory CD8(+) T cell responses to cancer. Semin Immunol 49:101435. https://doi.org/ 10.1016/j.smim.2020.101435

Helbig D, Quaas A, Mauch C et al (2017) Copy number variations in atypical fibroxanthomas and pleomorphic dermal sarcomas. Oncotarget 8:109457-109467

Hieronymus H, Murali R, Tin A et al (2018) Tumor copy number alteration burden is a pan-cancer prognostic factor associated with recurrence and death. Elife 7:e37294. https://doi.org/10.7554/ eLife.37294

Liu TT, Achrol AS, Mitchell LA et al (2016) Computational identification of tumor anatomic location associated with survival in 2 large cohorts of human primary glioblastomas. AJNR Am J Neuroradiol 37:621-628. https://doi.org/10.3174/ajnr.A4631

Liu L, Bai X, Wang J et al (2019) Combination of TMB and CNA stratifies prognostic and predictive responses to immunotherapy across metastatic cancer. Clin Cancer Res 25:7413-7423. https:// doi.org/10.1158/1078-0432.CCR-19-0558

Lopez-Campistrous A, Adewuyi EE, Williams DC et al (2020) Gene expression profile of epithelial-mesenchymal transition mediators in papillary thyroid cancer. Endocrine. https://doi.org/10.1007/ s12020-020-02466-3

Moon H, Donahue LR, Choi E et al (2017) Melanocyte stem cell activation and translocation initiate cutaneous melanoma in response to UV exposure. Cell Stem Cell 21(665-678):e666. https://doi. org/10.1016/j.stem.2017.09.001

Motomura K, Natsume A, Watanabe R et al (2012) Immunohistochemical analysis-based proteomic subclassification of newly diagnosed glioblastomas. Cancer Sci 103:1871-1879. https:// doi.org/10.1111/j.1349-7006.2012.02377.x

Mueller AA, van Velthoven CT, Fukumoto KD et al (2016) Intronic polyadenylation of PDGFRalpha in resident stem cells attenuates muscle fibrosis. Nature 540:276-279. https://doi.org/10. 1038/nature20160

Neftel C, Laffy J, Filbin MG et al (2019) An integrative model of cellular states, plasticity, and genetics for glioblastoma. Cell 178(835-849):e821. https://doi.org/10.1016/j.cell.2019.06.024

Ong HS, Gokavarapu S, Tian Z et al (2018) PDGFRA mRNA overexpression is associated with regional metastasis and reduced survival in oral squamous cell carcinoma. J Oral Pathol Med 47:652-659. https://doi.org/10.1111/jop.12713

Pantaleo MA, Tarantino G, Agostinelli C et al (2019) Immune microenvironment profiling of gastrointestinal stromal tumors (GIST) shows gene expression patterns associated to immune checkpoint inhibitors response. Oncoimmunology 8:e1617588. https://doi.org/10.1080/2162402X.2019.1617588

Paugh BS, Broniscer A, Qu C et al (2011) Genome-wide analyses identify recurrent amplifications of receptor tyrosine kinases and cell-cycle regulatory genes in diffuse intrinsic pontine glioma. J Clin Oncol 29:3999-4006. https://doi.org/10.1200/ JCO.2011.35.5677

Phillips JJ, Aranda D, Ellison DW et al (2013) PDGFRA amplification is common in pediatric and adult high-grade astrocytomas and identifies a poor prognostic group in IDH1 mutant glioblastoma. Brain Pathol 23:565-573. https://doi.org/10.1111/bpa.12043

Pozniak J, Nsengimana J, Laye JP et al (2019) Genetic and environmental determinants of immune response to cutaneous melanoma. Cancer Res 79:2684-2696. https://doi.org/10.1158/0008-5472. CAN-18-2864

Schiefer AI, Mesteri I, Berghoff AS et al (2015) Evaluation of tyrosine kinase receptors in brain metastases of clear cell renal cell carcinoma reveals cMet as a negative prognostic factor. Histopathology 67:799-805. https://doi.org/10.1111/his.12709

Seo JS, Ju YS, Lee WC et al (2012) The transcriptional landscape and mutational profile of lung adenocarcinoma. Genome Res 22:2109-2119. https://doi.org/10.1101/gr.145144.112

Seo N, Shirakura Y, Tahara Y et al (2018) Activated CD8(+) T cell extracellular vesicles prevent tumour progression by targeting of lesional mesenchymal cells. Nat Commun 9:435. https://doi.org/ 10.1038/s41467-018-02865-1

Shao X, Lv N, Liao J et al (2019) Copy number variation is highly correlated with differential gene expression: a pan-cancer study. BMC Med Genet 20:175. https://doi.org/10.1186/s12881-019-0909-5

Silva S, Danson S, Teare D et al (2018) Genome-wide analysis of circulating cell-free DNA copy number detects active melanoma and predicts survival. Clin Chem 64:1338-1346. https://doi.org/ 10.1373/clinchem.2018.290023 
Sokol ES, Pavlick D, Khiabanian H et al (2020) Pan-cancer analysis of BRCA1 and BRCA2 genomic alterations and their association with genomic instability as measured by genome-wide loss of heterozygosity. JCO Precis Oncol 4:442-465. https://doi.org/10. 1200/po.19.00345

Terada T (2012) Protein expression and gene mutation status of KIT and PDGFRA in renal cell carcinoma. Histol Histopathol 27(297):302

Thorsson V, Gibbs DL, Brown SD et al (2018) The immune landscape of cancer. Immunity 48(812-830):e814. https://doi.org/10.1016/j. immuni.2018.03.023

Vasudevaraja V, Rodriguez JH, Pelorosso C et al (2021) Somatic focal copy number gains of noncoding regions of receptor tyrosine kinase genes in treatment-resistant epilepsy. J Neuropathol Exp Neurol 80:160-168. https://doi.org/10.1093/jnen/nlaa137

Wang J, Cui R, Clement CG et al (2020a) Activation PDGFR-alpha/ AKT mediated signaling pathways in oral squamous cell carcinoma by mesenchymal stem/stromal cells promotes anti-apoptosis and decreased sensitivity to cisplatin. Front Oncol 10:552. https:// doi.org/10.3389/fonc.2020.00552

Wang Y, Li L, Yang Y et al (2020b) Copy number variation in MUC5AC and susceptibility to allergic rhinitis: a low-coverage whole-genome sequencing and validation cohort study. Genet Test Mol Biomark 24:173-180. https://doi.org/10.1089/gtmb. 2019.0166
Wang Y, Ren F, Song Z et al (2020c) Multiomics profile and prognostic gene signature of $\mathrm{m} 6 \mathrm{~A}$ regulators in uterine corpus endometrial carcinoma. J Cancer 11:6390-6401. https://doi.org/10.7150/jca. 46386

Yuan H, Yan M, Zhang G et al (2019) CancerSEA: a cancer single-cell state atlas. Nucleic Acids Res 47:D900-D908. https://doi.org/10. 1093/nar/gky939

Zarghooni M, Bartels U, Lee E et al (2010) Whole-genome profiling of pediatric diffuse intrinsic pontine gliomas highlights plateletderived growth factor receptor alpha and poly (ADP-ribose) polymerase as potential therapeutic targets. J Clin Oncol 28:13371344. https://doi.org/10.1200/JCO.2009.25.5463

Zhao M, Liu Y, Qu H (2016) Expression of epithelial-mesenchymal transition-related genes increases with copy number in multiple cancer types. Oncotarget 7:24688-24699

Zheng X, Amos CI, Frost HR (2020) Cancer prognosis prediction using somatic point mutation and copy number variation data: a comparison of gene-level and pathway-based models. BMC Bioinform 21:467. https://doi.org/10.1186/s12859-020-03791-0

Publisher's Note Springer Nature remains neutral with regard to jurisdictional claims in published maps and institutional affiliations. 